\title{
Cardiovascular manifestations of subarachnoid haemorrhage
}

\author{
Sethuraman Manikandan
}

\begin{abstract}
Subarachnoid haemorrhage (SAH) is one of the devastating conditions, especially the aneurysmal bleed which has high mortality as well as morbidity. The mortality and morbidity caused by SAH have been recognised to be caused by both neurological and systemic causes. The alterations in systemic and other organ damage could cause death in up to $40 \%$ of $\mathrm{SAH}$ patients.Among the systemic manifestations, cardiovascular and respiratory complications increase hospitalisation and worsen the outcome.The main pathophysiological mechanism is the increased sympathetic activation causing myocardial necrosis.Various cardiovascular manifestations range from electrocardiogram changes to myocardial ischaemia, cardiac failure and arrhythmias. This review deals with the cardiac manifestations in SAH patients.
\end{abstract}

Key words: Cardiac enzymes, electrocardiographic changes, neurogenic stunned myocardium, subarachnoid haemorrhage

\section{INTRODUCTION}

Subarachnoid haemorrhage (SAH) is one of the devastating conditions, especially aneurysmal bleed with high mortality as well as morbidity. The estimated incidence of aneurysmal SAH is approximately 5-20/1 lac population. ${ }^{[1]}$ The mortality and morbidity caused by SAH have been recognised to be caused by both neurological and systemic causes. The alterations in systemic and other organ damage could cause death in up to $40 \%$ of SAH patients..$^{[2,3]}$

Cardiac, pulmonary, inflammatory and renal effects constitute the major extracranial organ dysfunction

Professor and Head, Divison of Neuroanesthesia and Neurocritical Care, Sree Chitra Tirunal Institute for Medical Sciences and Technology, Thiruvananthapuram, Kerala, India

Address for correspondence:

Dr. Sethuraman Manikandan, A-4, NFQ, Sree Chitra Tirunal Institute for Medical Sciences and Technology, Poonthi Road, Kumarapuram, Trivandrum - 695 011, Kerala, India.

E-mail: kanmanisethu@gmail.com

\begin{tabular}{|l|l|}
\hline \multicolumn{2}{|c|}{ Access this article online } \\
\hline Quick Response Code: & Website: \\
\hline & www.jnaccjournal.org \\
\cline { 2 - 2 } & \\
\hline & \\
\hline
\end{tabular}

in these patients. ${ }^{[4]}$ This chapter specifically attempts to describe the cardiovascular manifestations of aneurysmal SAH. The importance for anaesthesiologist lies in the differentiation of secondary myocardial damage caused by SAH with the primary cardiac disease.

\section{PATHOPHYSIOLOGY}

The exact mechanism by which the systemic manifestations occur in SAH is unknown. However, it has been postulated that acute SAH triggers widespread neuroendocrine responses, inflammatory and immune-mediated mechanisms that are responsible for its systemic actions including the myocardium and the blood vessels. Various neural and humoral mechanisms have been implicated in the cardiac dysfunction in SAH. Immediately following SAH, there is intense activation in the regions of hypothalamus, insula and brain stem signifying activation of sympathetic nerve endings causing release of norepinephrine. ${ }^{[5,6]}$

This is an open access article distributed under the terms of the Creative Commons Attribution-NonCommercial-ShareAlike 3.0 License, which allows others to remix, tweak, and build upon the work non-commercially, as long as the author is credited and the new creations are licensed under the identical terms.

For reprints contact: reprints@medknow.com

How to cite this article: Manikandan S. Cardiovascular manifestations of subarachnoid haemorrhage. J Neuroanaesthesiol Crit Care 2017;4:S38-44. 
Blocking of cardiac sympathetic nerves has been found to reduce the cardiac injury in animal experiments. In addition, it has been found that there are high levels of circulating catecholamines in the blood of patients with cardiac damage indicating the stimulation of adrenal medulla and the humoral mechanism of injury. ${ }^{[7,8]}$

The histopathology of myocardial necrosis is called coagulative myocytolysis and is characterised by an excessive calcium influx and early myocyte calcification. ${ }^{[9,10]}$ In addition, an influx of neutrophil granulocytes, lymphocytes and macrophages occurs into the myocardium of patients who died after SAH. In some, this feature coincided with myocytolysis and thrombi in intramyocardial arteries. This may pave the way for a novel therapeutic option in the management like antithrombotic agents. ${ }^{[11]}$

\section{INFLAMMATORY CASCADE}

In addition to the above mechanisms, inflammatory cascade also has been postulated in the development of cardiac injury caused by activation of the monocytes release of inflammatory cytokines.

Neuroinflammation is another potential mechanism contributing to the injury to the brain and heart in both acute and delayed phase of SAH. Various therapeutic modalities are being tried to reduce the neuroinflammatory response..$^{[12]}$ Immediately following $\mathrm{SAH}$, there is disruption of blood brain barrier and release of blood in subarachnoid space. The red blood cells disintegrate to haemoglobin, haeme and haemin. The haemin is considered to release redox-active iron which tilts the balance between antioxidants (NADPH and glutathione) versus oxidants (superoxide, liposomal peroxidation products). To remove the haemoglobin from subarachnoid space, microglia cells are activated which attracts the neutrophils and macrophages from blood vessels. The neutrophils and macrophages are recruited from the blood into the subarachnoid space and get trapped in the subarachnoid space. Their activation as well as death causes release of inflammatory mediators such as cytokines, complement activation which are thought to be responsible for vasospasm and delayed cerebral ischaemia (DIC), meningitis, etc. Interleukin 1 (IL-1), IL-6 and tumour necrosis factor are released into both the serum and cerebrospinal fluid following SAH. The role of cytokines is yet to be elucidated; the clinical signs such as neutrophilia, pyrexia and general cerebral oedema are thought to be associated with the cytokine storm. The magnitude of inflammation affects the outcome of the patients in SAH. In addition to the local inflammatory reaction, the systemic inflammatory reactions to SAH have been also thought to be contributing to the clinical features.
Following $\mathrm{SAH}$, there is a decrease in parasympathetic outflow and increased sympathetic activity caused by ischaemia to hypothalamus, temporal lobe and brain stem. This causes myocardial and endothelial damage in peripheral blood vessels. The damaged myocardial and endocardial cells also provoke the systemic inflammatory response, activation of cytokines, complement system. This activation reaches the brain through blood vessels and can further damage the brain causing ischaemic damage.

\section{CLINICAL MANIFESTATIONS}

The cardiac manifestations of SAH can be varied from asymptomatic to fulminant myocardial damage, cardiac failure and sudden cardiac arrest. The true incidence of the various cardiac anomalies in SAH is not known due to lack of appreciation of the cardiac dysfunction in SAH patients, especially in mild forms, under-reporting, presence of coexisting cardiac diseases with SAH and specific test used for diagnosing the myocardial damage in SAH patients. The changes in the heart available in the literature in SAH patients are described below.

\section{Electrocardiographic changes}

Electrocardiographic (ECG) changes are the most widely recognised and studied abnormalities following SAH. ECG changes are seen in $25 \%-90 \%$ of SAH patients. ${ }^{[13-15]}$ Burch et al. described the repolarization abnormalities seen in stroke and SAH as 'cerebral T waves' as early as $1954 .{ }^{[16]}$

ECG changes are usually seen in the acute stage of SAH and resolve within 6 weeks. The ECG changes can be either repolarization abnormalities or ischaemic changes. In addition, rhythm and conduction disturbances also may occur alone or coexist with other ECG changes. Majority of the rhythm changes are benign and include sinus bradycardia or sinus tachycardia. Atrial fibrillation, ventricular ectopics, junctional rhythm have also been reported to occur in SAH patients. ${ }^{[17]}$ Predisposing factors for arrhythmia occurrence include female gender, QTc prolongation; myocardial damage caused by excessive sympathetic discharge, coronary vasospasm and electrolyte disturbances, pre-existing hypertension, etc.

Repolarization abnormalities are the most common ECG alterations described in patients with SAH. The common abnormalities seen are prolongation of the QT interval, changes in the ST segment and T wave morphology. The aetiology has been thought to be increased sympathetic activity and most likely due to right insular injury. ${ }^{[18]}$

Even though wide variety of ECG ischaemic changes such as ST segment elevation or inversion, pathological $\mathrm{Q}$ waves, $\mathrm{T}$ inversion, $\mathrm{U}$ waves are seen in $\mathrm{SAH}$ patients, the presence of either inverted $\mathrm{T}$ waves or severe 
QTc segment prolongation on any ECG change was associated with $100 \%$ sensitivity and $81 \%$ specificity for left ventricular (LV) dysfunction determined as regional wall motion abnormalities (RWMAs) in echocardiography. Borderline creatine kinase-MB elevation $(\geq 2 \%)$ was associated with a similar high degree of sensitivity $(100 \%)$ and specificity $(94 \%) \cdot{ }^{[19]}$ In another series of SAH patients, Junttila et al. found that heart rate increased, PQ and QRS interval decreased and was associated with QTc prolongation in 91\% SAH patients. Repolarization abnormalities were associated with female gender and propofol use whereas ischaemic ECG changes were seen in male gender. Both repolarization and ischaemic changes were associated with aneurysmal bleeding, increase in cardiac troponin I (cTnI). Ischaemic changes were associated with global LV dysfunction, whereas in repolarization changes, LV function was in the normal range. Repolarization abnormalities were not seen in low Glasgow Coma Scale patients who were intubated and sedated in their series indicating the sympathoadrenal mechanism of ECG changes. The ischaemic changes were associated with poor outcome whereas the repolarization abnormalities were not. ${ }^{[20]}$

In a study of the follow-up of IHAST trial, the admission ECG changes in the pre-operative period with $\mathrm{SAH}$ were analysed for correlation of ECG changes with the outcome. The analysis found that ECG changes were seen in $80 \%$ of SAH patients at admission. The most common abnormality seen in ECG was non-specific ST/T-wave changes (NSSTTWA), followed by ST elevation, T-wave inversion and ST depression. ECG abnormalities were most commonly observed in the anterior leads. There was no correlation between ECG changes and World ferderation of Neurosurgical Society (WFNS) score as well as Fisher grade of SAH severity. The study showed that pre-operative bradycardia (heart rate 60 beats/ min), relative tachycardia (heart rate 80 beats/min) and NSSTTWA were associated with increased mortality in patients with SAH treated with surgical aneurysm clipping. The researchers have suggested compared to pre-operative clinical status; ECG changes may have more prognostication value in 1-year outcome of SAH patients. In addition, they found that pre-operative NSSTTWA, relative tachycardia and a prolonged QTc interval identified patients who would have haemodynamic instability, need for vasopressors during their hospital stay. ${ }^{[21]}$ In addition to the effects of ECG on cardiac function, abnormal $Q$ or QS wave and NSSTTWA were independently associated with neurogenic pulmonary oedema (NPE). Their finding shows that NPE may be the result of myocardial injury caused by excessive sympathetic stimulation. ${ }^{[22]}$ Majority of ECG changes occurred within $24 \mathrm{~h}$, especially ischaemic changes representing the myocardial injury. The QTC prolongation can be delayed up to $48 \mathrm{~h} .{ }^{[21]}$
In a study trying to correlate ECG changes with DIC, no association was found between ECG changes and the incidence of DCI. ${ }^{[23]}$ However, a recent study from the CONSCIOUS-1 trial has shown that QTc prolongation and sinus tachycardia were associated with increased incidence of vasospasm. ${ }^{[24]}$

\section{Echocardiographic changes}

Echocardiography is a major diagnostic tool in patients with aneurysmal SAH in assessing the cardiac function and has been studied more elaborately. Changes in cardiac function due to SAH using echocardiography have been well recognised. Echocardiography may show features of global or regional systolic dysfunction, diastolic dysfunction. There can be fallen in stroke volume and cardiac output. The incidence of impaired LV function in the form of either RWMAs or globally impaired contractility by echocardiography ranged from $8 \%$ to $50 \% \cdot{ }^{[25,26]}$

In a small series of SAH patients, ECG changes were seen in $48 \%$ of patients whereas echo evidence of RWMA was found in $9 \%$. All the patients with echo abnormality had ECG changes of ischaemia. The echo changes correlated with the SAH severity grade whereas no such correlation was found with respect to ECG changes. Moreover, the researchers have found that echo changes were seen predominantly in anterior circulation aneurysms whereas ECG changes were seen more in posterior circulation aneurysms. ${ }^{[27]}$

RWMA was seen to occur within 1-2 days of SAH and gradually improves over time. Majority of patients with RWMA following SAH have normal coronaries. Pre-operative comorbidities such as hypertension, old age, diabetes mellitus and hyperlipidaemia were not associated with risk of RWMA whereas poor Hunt-Hess classification, elevated cTnI $>1.0 \mu \mathrm{g} / \mathrm{L}$, has been found to be a strong predictor of RWMA. ${ }^{[28]}$ RWMA has been found to occur at either admission or later during hospitalization. Abnormal findings on admission ECG (sinus tachycardia, ST-segment elevation or ST-segment depression) and positive troponin $\mathrm{T}$ are risk factors for early RWMA, and a myocardial infarction (MI) pattern on the admission ECG and positive troponin T also predicts late RWMA. ${ }^{[29]}$ Studies have shown that the RWMA is seen in the apical segments whereas the basal segments were hyperkinetic. Most of the RWMA also occurs in the anterior or anteroseptal area. However, some studies have shown that RWMA does not correspond to a particular coronary artery territory. RWMA has been associated with poor outcome including DIC and death. Patients with apical RWMAs had more risk of DCI, whereas mid-ventricular WMAs were associated with death. This finding might be explained by an impaired cardiac output in combination 
with disturbed cerebral autoregulation after aneurysmal SAH. ${ }^{[30]}$ In another study, the same group has found that patient with LV dysfunction had lower cerebral blood flow compared to those without LV dysfunction. ${ }^{[31]}$

In addition to the global and regional systolic function, the right ventricular function may be impaired in $\mathrm{SAH}$ patients. Diastolic function is also affected. Impaired diastolic function was seen in $71 \%$ of SAH patients and was associated with the development of pulmonary oedema and elevated troponin $\mathrm{T}$ levels. Diastolic dysfunction can impair the fluid management in these patients. ${ }^{[32]}$

\section{Neurogenic stunned myocardium and} stress-induced (Takotsubo) cardiomyopathy Besides the ECG changes and RWMA, extensive myocardial injury following SAH can present as severely depressed global cardiac, especially LV function. The condition is described as neurogenic stunned myocardium (NSM). The condition is considered as fully reversible, occurring within $24 \mathrm{~h}$ of SAH and gradual recovery as early as $48 \mathrm{~h}$ to 7 days. Recent terminology for NSM is 'stress-related cardiomyopathy syndromes'. ${ }^{[33]}$ In addition, the patient may present as stress-induced cardiomyopathy or takotsubo cardiomyopathy (TC). The proposed diagnostic criteria for NSM are as follows: ${ }^{[34]}$

- Acute structural or functional brain disorder

- New onset of systolic and/or diastolic LV dysfunction. Systolic dysfunction can include RWMA and/or global wall-motion abnormality. RWMAs should be extending beyond a single epicardial vascular distribution

- Partial or complete resolution of LV dysfunction in 4 weeks

- At least one of the following:

a. No history of congestive heart failure, LV dysfunction or coronary artery disease

b. No evidence of myocardial ischaemia on myocardial perfusion scan

c. Absence of angiographic evidence of obstructive coronary disease or of acute plaque rupture.

The risk factors for NSM development include Hess and Hunt SAH grade of $>3$, old age, history of smoking. Other factors were not found to be significant in the development of NSM. Variations in NSM can also occur in SAH which consists of: ${ }^{[3]}$

- Apical and mid-ventricular LV dysfunction (TC)

- Isolated mid-ventricular and basal LV dysfunction/ isolated mid-ventricular LV dysfunction (apical-sparing TC)

- Isolated basal LV dysfunction

- Global LV hypokinesia

- Other non-coronary distribution wall motion abnormalities.
TC or broken heart syndrome is a severe form of myocardial injury following SAH. It is described in post-menopausal women and with a variety of stress such as surgery, $\mathrm{SAH}$, head injury and emotional stress. The clinical presentation resembles that of acute coronary syndrome (ACS) like chest pain, ischaemic changes in ECG and elevation of cardiac enzymes. The characteristic echo findings are apical ballooning with RWMA of apical and mid-segments, hyper contracting basal regions.

Mayo Clinic has proposed diagnostic criteria for TC in 2004, which have been modified recently: ${ }^{[35]}$ (1) transient hypokinesis, akinesis or dyskinesis in the LV mid-segments with or without apical involvement; RWMAs that extend beyond a single epicardial vascular distribution; and frequently, but not always, a stressful trigger; (2) the absence of obstructive coronary disease or angiographic evidence of acute plaque rupture; (3) new ECG abnormalities (ST-segment elevation and/or T-wave inversion) or modest elevation in cTn; and (4) the absence of pheochromocytoma and myocarditis.

Takotsubo cardiomyopathy must be differentiated from ACS. Elevation of CTnI levels in TC are not as high as ACS and there is relative sparing of basal regions and angiographically normal coronary arteries compared to ACS. TC needs to be differentiated from NSM. TC presents with features of chest pain, ECG shows ST elevation whereas NSM presents with heart failure, T wave inversion and no ST changes are seen in ECG. Most TC patients present with the typical apical ballooning due to mid-ventricular and apical a/dyskinesia with hypercontractile basal segments while NS patients showed hypo/akinesia affecting predominantly the basal and mid-ventricular segments. Cardiac enzyme levels were elevated similarly. However, TC represents transmural ischaemia and is more severe than NSM which causes subendocardial ischaemia. ${ }^{[36]}$

\section{Cardiac enzymes elevation}

Approximately $30 \%-50 \%$ of SAH patients who reach the hospital alive die and another $10 \%-20 \%$ remain dependent on help for daily activities. It is of paramount importance to differentiate the myocardial dysfunction from NSM following SAH. Bulsara et al. retrospectively analysed the SAH patients admitted to their hospital from 1995 to 2000 using ECG, echocardiography, creatine phosphokinase (CPK)-MB and cTn. They found that the NSM occurred in more severe grades of SAH. The CPK-MB and ECG did not correlate with NSM. The cTnI increased within $12 \mathrm{~h}$, peaked within $48 \mathrm{~h}$ and returned to normal in 7-10 days. cTnI is a regulatory protein which is highly specific for cardiac muscle. It is considered to be a reliable marker of myocardial injury leading to LV dysfunction. A raised cTnI concentration is a marker of poor prognosis in patients with unstable cardiac ischaemia and in patients with 
septic shock. To differentiate MI from SM following $\mathrm{SAH}$, the authors suggested the following criteria; based on our findings, we propose the following criteria to differentiate acute MI from reversible SM associated with aneurysmal SAH: (1) No history of cardiac problems; (2) new onset of abnormal cardiac function (ejection fraction [EF] - 40\%); (3) cardiac wall motion abnormalities on echocardiogram that do not correlate with the coronary vascular distribution noted on ECGs; and (4) cTn values $<2.8 \mathrm{ng} / \mathrm{ml}$ in patients with an $\mathrm{EF}<40 \%(>2.8 \mathrm{ng} / \mathrm{ml}$ strongly suggestive of $\mathrm{MI}) .{ }^{[37]}$

Studies have shown that in $30 \%-40 \%$ of patients with $\mathrm{SAH}$, increased concentrations of $\mathrm{cTnI}$ were seen on admission. Increased cTnI proved to be a highly sensitive and specific marker for cardiac dysfunction in patients with SAH too. ${ }^{[38]}$ Studies assessing the level of cTnI on the prognostication have shown variable levels. This is due to the fact the cut-off level of the enzyme used for the assessment. ${ }^{[39]}$ However, Schuiling et al. have found a cut-off level of cTnI of. $0.3 \mu \mathrm{g} / \mathrm{L}$ found to be significant for cardiac dysfunction in SAH. The elevated enzyme level correlated with pulmonary oedema, disturbances of pulmonary gas exchange, rhythm disturbances and an inadequate cardiac performance. Patients with elevated enzymes also had a poor outcome. Moreover, the severity of increase correlated with the severity of SAH. ${ }^{[40]}$

In a similar study, Tanabe et al. showed that cTnI levels of $>1.3 \mathrm{ng} / \mathrm{ml}$ were associated RWMAs with a sensitivity of $76 \%$ and specificity of $91 \%$. The anterior and anteroseptal areas were showing RWMA and last for a week from the onset of SAH. This correlated with the decrease in the level of cTnI. Moreover, the researchers have found that $79 \%$ of patients with levels $>0.1 \mathrm{ng} / \mathrm{ml}$ were associated with pulmonary congestion, diastolic dysfunction and elevated LV filling pressures. A highly positive cTnI was associated with severity of neurologic damage and longer intensive care unit stay, as well as evidence of global and regional LV systolic dysfunction, although degrees of EF depression and wall motion abnormalities were typically modest and transient. Mild increases in cTnI were associated with diastolic dysfunction, and importantly, the occurrence of pulmonary congestion increased with $\mathrm{CTnI} .{ }^{[41]}$

Serum plasma B-type natriuretic peptide (BNP) is a global indicator of left cardiac dysfunction. Stretch of cardiac myocytes is a potent stimulus for BNP secretion. BNP levels have been shown to increase in conditions such as diastolic dysfunction and acute heart failure. Serum BNP levels also have been shown to increase following SAH, especially in NPE and myocardial dysfunction. The elevated levels are seen within $48 \mathrm{~h}$ of aneurysm rupture and last for 2-3 weeks. The average increase in serum levels of the enzyme appears to be 2-3 times normal values. In a series of SAH patients, $80 \%$ of patients develop a BNP levels of $>100 \mathrm{ng} / \mathrm{L}$ during the first 3 days (peak on day 2 ) and lasted for 1 week. ${ }^{[42]}$ The levels of BNP did not correlate with severity of SAH grade or can be used as a prognostic of the mortality or DIC. The time course of change in the levels of BNP along with cTnI gives a useful guide in the recovery of myocardial function. A cTnI $>89 \mathrm{ng} / \mathrm{L}$ on day 1 after onset of symptoms, a proBNP $>2615 \mathrm{ng} / \mathrm{L}$ on day 2 after onset of symptoms and a proBNP $>1830 \mathrm{ng} / \mathrm{L}$ on day 3 after onset of symptoms had best values for detection of myocardial injury following SAH, respectively, with a sensitivity of $100 \%$ and specificity of $83 \%-91 \%{ }^{[43]}$

\section{CLINICAL RELEVANCE FOR ANAESTHESIOLOGIST}

Recognition of the presence of myocardial dysfunction is very important in the perioperative and critical care management of aneurysmal SAH patients as it influences the outcome. In addition, it is important to assess the severity of myocardial dysfunction which helps in modifying treatment. This is usually accomplished with ECG, echocardiography and cardiac enzymes monitoring. Since the dysfunction is reversible, early recognition will help differentiate from ACS and prevent unnecessary further tests or coronary bypass. In addition, it helps in timing for either intervention or surgery as the NSM usually resolves in 10 days. In the presence of diastolic dysfunction or systolic dysfunction or pulmonary oedema, it will be important to use intravenous fluids and inotropes judiciously. There are limited studies or guidelines in the management of these patients, and the management needs to be titrated individually.

\section{Financial support and sponsorship}

Nil.

\section{Conflicts of interest}

There are no conflicts of interest.

\section{REFERENCES}

1. Ingall $\mathrm{T}$, Asplund $\mathrm{K}$, Mähönen $\mathrm{M}$, Bonita R. A multinational comparison of subarachnoid hemorrhage epidemiology in the WHO MONICA stroke study. Stroke 2000;31:1054-61.

2. Kassell NF, Torner JC, Haley EC Jr., Jane JA, Adams HP, Kongable GL. The international cooperative study on the timing of aneurysm surgery. Part 1: Overall management results. J Neurosurg 1990;73:18-36.

3. Solenski NJ, Haley ECJr., Kassell NF, Kongable G, Germanson T, Truskowski L, et al. Medical complications of aneurysmal subarachnoid hemorrhage: A report of the multicenter, cooperative aneurysm study. Participants of the multicenter cooperative aneurysm study. Crit Care Med 1995;23:1007-17.

4. Gruber A, Reinprecht A, Illievich UM, Fitzgerald R, Dietrich W, Czech T, et al. Extracerebral organ dysfunction and neurologic outcome after aneurysmal subarachnoid hemorrhage. Crit Care Med 1999;27:505-14.

5. Doshi R, Neil-Dwyer G. A clinicopathological study of 
patients following a subarachnoid hemorrhage. J Neurosurg 1980;52:295-301.

6. Doshi R, Neil-Dwyer G. Hypothalamic and myocardial lesions after subarachnoid haemorrhage. J Neurol Neurosurg Psychiatry 1977;40:821-6.

7. Masuda T, Sato K, Yamamoto S, Matsuyama N, Shimohama T, Matsunaga A, et al. Sympathetic nervous activity and myocardial damage immediately after subarachnoid hemorrhage in a unique animal model. Stroke 2002;33:1671-6.

8. Naredi S, Lambert G, Eden E, Zall S, Runnerstam M, Rydenhag B, et al. Increased sympathetic nervous activity in patients with non-traumatic subarachnoid hemorrhage. Stroke 2000;31:901-6.

9. Uchida Y, Egami H, Uchida Y, Sakurai T, Kanai M, Shirai S, et al. Possible participation of endothelial cell apoptosis of coronary microvessels in the genesis of takotsubo cardiomyopathy. Clin Cardiol 2010;33:371-7.

10. Mann DL, Kent RL, Parsons B, Cooper G $4^{\text {th }}$. Adrenergic effects on the biology of the adult mammalian cardiocyte. Circulation 1992;85:790-804.

11. van der Bilt IA, Vendeville JP, van de Hoef TP, Begieneman MP, Lagrand WK, Kros JM, et al. Myocarditis in patients with subarachnoid hemorrhage: A histopathologic study. J Crit Care 2016;32:196-200.

12. Lucke-Wold BP, Logsdon AF, Manoranjan B, Turner RC, McConnell E, Vates GE, et al. Aneurysmal subarachnoid hemorrhage and neuroinflammation: A comprehensive review. Int J Mol Sci 2016;17:497.

13. Brouwers PJ, Wijdicks EF, Hasan D, Vermeulen $M$, Wever EF, Frericks $\mathrm{H}$, et al. Serial electrocardiographic recording in aneurysmal subarachnoid hemorrhage. Stroke 1989;20:1162-7.

14. Zaroff JG, Rordorf GA, Newell JB, Ogilvy CS, Levinson JR. Cardiac outcome in patients with subarachnoid hemorrhage and electrocardiographic abnormalities. Neurosurgery 1999;44:34-9.

15. Sommargren CE, Zaroff JG, Banki N, Drew BJ. Electrocardiographic repolarization abnormalities in subarachnoid hemorrhage. J Electrocardiol 2002;35 Suppl:257-62.

16. Burch GE, Meyers R, Abildskov JA. A new electrocardiographic pattern observed in cerebrovascular accidents. Circulation 1954;9:719-23.

17. Katsanos AH, Korantzopoulos P, Tsivgoulis G, Kyritsis AP, Kosmidou M, Giannopoulos S. Electrocardiographic abnormalities and cardiac arrhythmias in structural brain lesions. Int J Cardiol 2013;167:328-34.

18. Sakr YL, Lim N, Amaral AC, Ghosn I, Carvalho FB, Renard M, et al. Relation of ECG changes to neurological outcome in patients with aneurysmal subarachnoid hemorrhage. Int J Cardiol 2004;96:369-73.

19. Mayer SA, LiMandri G, Sherman D, Lennihan L, Fink ME, Solomon RA, et al. Electrocardiographic markers of abnormal left ventricular wall motion in acute subarachnoid hemorrhage. J Neurosurg 1995;83:889-96.

20. Junttila E, Vaara M, Koskenkari J, Ohtonen P, Karttunen A, Raatikainen $\mathrm{P}$, et al. Repolarization abnormalities in patients with subarachnoid and intracerebral hemorrhage: Predisposing factors and association with outcome. Anesth Analg 2013;116:190-7.

21. Coghlan LA, Hindman BJ, Bayman EO, Banki NM, Gelb AW, Todd MM, et al. Independent associations between electrocardiographic abnormalities and outcomes in patients with aneurysmal subarachnoid hemorrhage: Findings from the intraoperative hypothermia aneurysm surgery trial. Stroke 2009;40:412-8.

22. Chen WL, Huang $\mathrm{CH}$, Chen JH, Tai HC, Chang SH, Wang YC.
ECG abnormalities predict neurogenic pulmonary edema in patients with subarachnoid hemorrhage. Am J Emerg Med 2016;34:79-82.

23. Schuiling WJ, Algra A, de Weerd AW, Leemans P, Rinkel GJ. ECG abnormalities in predicting secondary cerebral ischemia after subarachnoid haemorrhage. Acta Neurochir (Wien) 2006;148:853-8.

24. Ibrahim GM, Macdonald RL. Electrocardiographic changes predict angiographic vasospasm after aneurysmal subarachnoid hemorrhage. Stroke 2012;43:2102-7.

25. Pollick C, Cujec B, Parker S, Tator C. Left ventricular wall motion abnormalities in subarachnoid hemorrhage: An echocardiographic study. J Am Coll Cardiol 1988;12:600-5.

26. ZaroffJG, Rordorf GA, Ogilvy CS, Picard MH. Regional patterns of left ventricular systolic dysfunction after subarachnoid hemorrhage: Evidence for neurally mediated cardiac injury. J Am Soc Echocardiogr 2000;13:774-9.

27. Davies KR, Gelb AW, Manninen PH, Boughner DR, Bisnaire D. Cardiac function in aneurysmal subarachnoid haemorrhage: A study of electrocardiographic and echocardiographic abnormalities. Br J Anaesth 1991;67:58-63.

28. Kothavale A, Banki NM, Kopelnik A, Yarlagadda S, Lawton MT, Ko N, et al. Predictors of left ventricular regional wall motion abnormalities after subarachnoid hemorrhage. Neurocrit Care 2006;4:199-205.

29. van der Bilt IA, Hasan D, van den Brink RB, Cramer MJ, van der Jagt M, van Kooten F, et al. Time course and risk factors for myocardial dysfunction after aneurysmal subarachnoid hemorrhage. Neurosurgery 2015;76:700-5.

30. van der Bilt I, Hasan D, van den Brink R, Cramer MJ, van der Jagt $\mathrm{M}$, van Kooten $\mathrm{F}$, et al. Cardiac dysfunction after aneurysmal subarachnoid hemorrhage: Relationship with outcome. Neurology 2014;82:351-8.

31. Cremers CH, van der Bilt IA, van der Schaaf IC, Vergouwen MD, Dankbaar JW, Cramer MJ, et al. Relationship between cardiac dysfunction and cerebral perfusion in patients with aneurysmal subarachnoid hemorrhage. Neurocrit Care 2016;24:202-6.

32. Kopelnik A, Fisher L, Miss JC, Banki N, Tung P, Lawton MT, et al. Prevalence and implications of diastolic dysfunction after subarachnoid hemorrhage. Neurocrit Care 2005;3:132-8.

33. Bybee KA, Prasad A. Stress-related cardiomyopathy syndromes. Circulation 2008;118:397-409.

34. Stevens RD, Nyquist PA. The systemic implications of aneurysmal subarachnoid hemorrhage. J Neurol Sci 2007;261:143-56.

35. Akashi YJ, Goldstein DS, Barbaro G, Ueyama T. Takotsubo cardiomyopathy: A new form of acute, reversible heart failure. Circulation 2008;118:2754-62.

36. Ancona F, Bertoldi LF, Ruggieri F, Cerri M, Magnoni M, Beretta L, et al. Takotsubo cardiomyopathy and neurogenic stunned myocardium: Similar albeit different. Eur Heart J 2016;37:2830-2.

37. Bulsara KR, McGirt MJ, Liao L, Villavicencio AT, Borel C, Alexander $\mathrm{MJ}$, et al. Use of the peak troponin value to differentiate myocardial infarction from reversible neurogenic left ventricular dysfunction associated with aneurysmal subarachnoid hemorrhage. J Neurosurg 2003;98:524-8.

38. Parekh N, Venkatesh B, Cross D, Leditschke A, Atherton J, Miles W, et al. Cardiac troponin I predicts myocardial dysfunction in aneurysmal subarachnoid hemorrhage. J Am Coll Cardiol 2000;36:1328-35.

39. Deibert E, Barzilai B, Braverman AC, Edwards DF, Aiyagari V, Dacey R, et al. Clinical significance of elevated troponin I levels in patients with nontraumatic subarachnoid hemorrhage. J Neurosurg 2003;98:741-6.

40. Schuiling WJ, Dennesen PJ, Tans JT, Kingma LM, Algra A, 
Rinkel GJ. Troponin I in predicting cardiac or pulmonary complications and outcome in subarachnoid haemorrhage. J Neurol Neurosurg Psychiatry 2005;76:1565-9.

41. Tanabe M, Crago EA, Suffoletto MS, Hravnak M, Frangiskakis JM, Kassam AB, et al. Relation of elevation in cardiac troponin I to clinical severity, cardiac dysfunction, and pulmonary congestion in patients with subarachnoid hemorrhage. Am J Cardiol 2008;102:1545-50.

42. Meaudre E, Jego C, Kenane N, Montcriol A, Boret $\mathrm{H}$,
Goutorbe P, et al. B-type natriuretic peptide release and left ventricular filling pressure assessed by echocardiographic study after subarachnoid hemorrhage: A prospective study in non-cardiac patients. Crit Care 2009;13:R76.

43. Oras J, Grivans C, Dalla K, Omerovic E, Rydenhag B, Ricksten SE, et al. High-sensitive troponin $\mathrm{T}$ and $\mathrm{N}$-terminal pro B-type natriuretic peptide for early detection of stress-induced cardiomyopathy in patients with subarachnoid hemorrhage. Neurocrit Care 2015;23:233-42. 